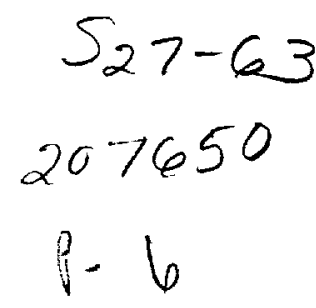

\title{
FUZZY LOGIC BASED ROBOTIC CONTROLLER
}

\author{
F. ATTIA* , M. UPADHYAYA** \\ * Associate Professor, ** Research Associate \\ University of Houston, College of Technology \\ 4800 Calhoun Road, Houston, Texas 77204 - 4083
}

\begin{abstract}
Existing Proportional-Integral-Derivative (PID) robotic controllers rely on an inverse kinematic model to convert user-specified cartesian trajectory coordinates to joint variables. These joints experience friction, stiction and gear backlash effects. Due to lack of proper linearization of these effects, modem control theory based on state space methods cannot provide adequate control for robotic systems. In presence of loads, the dynamic behavior of robotic systems is complex and nonlinear, especially where mathematical modeling is evaluated for real-time operations. Fuzzy Logic Control is a fast emerging alternative to conventional control systems in situations where it may not be feasible to formulate an analytical model of the complex system.
\end{abstract}

Fuzzy logic techniques track a user-defined trajectory without having the host computer to explicitly solve the nonlinear inverse kinematic equations. The goal is to provide a rule-based approach, which is closer to human reasoning. The approach used expresses endpoint error, location of manipulator joints, and proximity to obstacles as fuzzy variables. The resulting decisions are based upon linguistic and non-numerical information.

This paper presents a solution to the conventional robot controller which is independent of computationally intensive kinematic equations. Computer simulation results of this approach as obtained from software implementation are also discussed.

\section{Intreduction}

Fuzzy set theory was developed in 1965 by Zadeh [1], and permits the treatment of vague, uncertain, imprecise, and ill-defined knowledge and concepts in an exact mathematical way. This theory addresses the uncertainty that results from boundary conditions as opposed to Probability theory of mathematics. It allows one to express the operational and control laws of a system, linguistically in words such as "too cold", "cool", "warm", "very hot" etc., which is a generalization of the classical set theory. Fuzzy arithmetic differs from classical Boolean arithmetic as it allows a variable to be partially included in any given set as opposed to being fully included or excluded in Boolean algebra. This is known as Crisp set theory. Fuzzy logic is multivalued and varies from maximum to minimum as a function of the input. Fuzzy sets are subjective as compared to standard crisp sets which are objective and are viewed as exceptional cases of fuzzy sets [2].

Fuzzy controllers offer some practical advantages over conventional controllers like increased robustness in spite of high ambient noise levels or sensor failures, an ability to handle nonlinearities without control system degradation, and easy formulation of fuzzy rules. This makes the understanding, modification and maintenance of a fuzzy logic based controller much easier than is possible with conventional controllers. This method can be used when a specific rule base or expert is available who can specify the rules underlying the system behavior and the fuzzy set that represents the characteristics of each variable. The drawbacks of the inverse kinematic equations have posed significant limitations on the robot controller since it is difficult to move the end-effector to a specified position and computing joint variables.

This paper discusses a novel approach in designing a fuzzy logic controller for the robotic arm which replaces the traditional controller and lays the foundation for a new generation of robotic controllers with a simpler architecture.

\section{Conventional Controller Design of Manipulators}

The most common controller for robotic manipulators in feedback systems is the ProportionalIntegral-Derivative (PID) controller, which is implemented as a secondary controller. This controller corrects errors by means of trajectory tracking [3]. A PID controller performs Proportional amplification 
(P),Integration (I), and Differentiation (D) on the error signal fed into the controller as input. In general, the Dpart speeds up the response by performing a predictivetype function, I-part influences the steady-state error, and the P-part influences the open-loop steady-state gain. Each part of the controller needs adjustment or tuning experimentally so that desirable responses of the system are obtained. The gain of a PID controller can also be determined by Eigen value assignment.

The PID controller is very simple to implement and each axis can have its own separate PID loop. The main drawback of the PID controller is that the load seen by the motor or actuator of each joint can change rapidly and substantially. This is particularly true for the proximal joints near the base where the moments of inertia and the loading due to gravity can vary by an order of magnitude [4].

\section{Implementation of Euzzy Logic}

A fuzzy logic controller can be considered as a control expert system which simulates human thinking in the interpretation of the real world data. It utilizes fuzzy set labels and performs an appropriate reasoning using Compositional Rule of Inference (CRI) [5]. The CRI represents the core of the deduction mechanism of the controller. It performs the composition of fuzzy sets and matrices of fuzzy rules using the max-min operator. One of the main advantages of using fuzzy approach is that it provides the best technique for knowledge representation that could be possibly devised for encoding knowledge about continuous (analog) variables.

The components of the conventional and fuzzy systems are similar. They differ mainly in fuzzy systems containing the Fuzzifier which maps the input physical variables measured by an external sensor to fuzzy set variables [6]. The conditional rules expressed in the form of IF (some event) THEN (perform some action) are contained within the rule evaluator. The inverse process of converting the fuzzy outputs of the fuzzy rule evaluator to a physical variable is performed by the Defuzzifier. The value produced by the defuzzifier represents the weighted average of all fuzzy rules that were fired within the fuzzy rule evaluator.

The fuzzy system designer's task lies in defining the data points flowing in the system, the basic transformations performed on the data and the data elements output from the system. The first step consists of analyzing the system and understanding the given problem. Next, each control and solution variable in the fuzzy model is decomposed into a set of fuzzy regions. These regions are given unique names, called labels within the domain of the variable. The measured values of input are then converted to corresponding degrees of membership in fuzzy sets. This is done by applying the definition of membership functions for each input variable. Rules that tie the input values to the output model are written as follows : "if < fuzzy proposition $A>$, then < do fuzzy proposition B >". Generally, the number of rules a system requires is related to the number of control variables. The last step would be to select a method of "defuzzification". There are several ways to convert an output fuzzy set into a crisp solution variable, but the most commonly used one is the centroid technique. Thus the real complexity in developing a fuzzy system is in creating and testing both the degree of membership functions and the rule base, rather than implementing the run-time environment.

\section{Proposed Fuzzy Logic Controller Model}

The two basic problems encountered when attempting to apply a fuzzy control in real systems are:

- Choice of primary fuzzy sets to be used together with the rules that constitute the control law or algorithm for a fuzzy control structure.

- Numerical description of the linguistics to implement a fuzzy control algorithm in a computer, which is a nonfuzzy machine.

The typical robot control problem consists of moving the end-effector to a user-specified position $(x, y, z)$ and orientation (roll, pitch, yaw) [7]. To achieve this, the robot joint motors must be driven to specific angular positions. The task of computing these specific joint angles is referred to as the inverse kinematic problem. In general, inverse kinematic equations are highly coupled and involve nonlinear differential equations, whose closed form solutions are often undefined. This poses a computational bottleneck. The block diagram of the proposed Fuzzy Logic Controller is shown in Figure 1.

The Southwestern Research Institute (SWRI) [6] at San Antonio, Texas applied fuzzy logic to control a robot without having to explicitly solve inverse kinematic equations. This controller, mimics intelligent human-like decision-making via a fuzzy rule base, which is essentially a collection of varying degrees of causeand-effect relationships. The fuzzy rule base is the most critical element within the novel robot controller. The performance of the controller is directly dependent on the quality of fuzzy rules. The approach taken to realize the optimum set of rules which would track enabling control was to linearize the robot model and then apply the principle of superposition to the resulting linearized equations. First, the $\mathrm{x}$ and $\mathrm{y}$ components of the individual locations of robot joints and the observed tracking error of the robot end-effector need to be 
represented in fuzzy terms such as: Positive Big (PB), Positive Medium (PM) etc. up to Negative Big (NB). Next, simple fuzzy rules were formulated to evaluate the individual joint axis contributions to reduce the tracking errors of the robot end-effector. For example, if the tracking error in the $x$ direction is $P M$ and the $y$ component of the end-effector is $\mathrm{PB}$, then move the first joint by PM. If robot end point is Negative Medium (NM) and tracking error is Positive Big (PB), change joint angle 1 by NM.

\section{A Simple 2-Degree of Ereedom_Manipulator}

The problem of designing a manipulator controller stems from the basic idea of the simplest known biological controller which is the human arm [8]. When we reach for an object, we determine the approximate error (distance from our hand to the object), and move in a way to reduce the error. We do not precompute the path or the elbow or shoulder angles which is required to grasp the object. Our motions continuously aim at reducing the distance between the hand and the target. In fact, we are successful at reaching and grasping both stationary and moving objects and accomplish these feats without an accurate mathematical model of the kinematics involved. Thus, the fuzzy logic approach allows an initial control system to be derived from fundamental concepts without the need for extended training sets. There are several approaches that achieve this objective. One such approach is discussed in this paper.

The coordinates of the manipulator of the desired point, or target (the end-effector is assumed to be located at the tip of the second link, or at the second joint) are $\left(x_{d}, y_{d}\right),\left(x_{0}, y_{0}\right)$ the coordinates of the manipulator of the initial point, $e(r)$ is the error of the manipulator between the initial and the end points, $r_{d}$ and $\mathrm{r}_{0}$ are the desired and initial arm lengths (distance from the base joint to the manipulator), angles 180 C,180-D and $E$ are the initial and final angles between the links respectively and the error angle $E=C-D$, we have:

$$
\begin{aligned}
& e(r)^{2}=r_{d}^{2}-r_{0}^{2} \\
& =2 \cdot L 1 \cdot L 2 \cdot(\cos C-\sin C \cdot E-\cos C) \\
& \text { if angle } E \text { is small } \\
& =2 . \mathrm{L} 1 . \mathrm{L} 2 \operatorname{sinC} . \mathrm{E} \\
& \text { where } \sin E=E \text { for } E \ll 0 \text {. }
\end{aligned}
$$

Here, $e(r)^{2}$ is used as the input signal to the fuzzy set rules.

$$
\text { Actually, } e(r)^{2}=\left[\left(x_{d}{ }^{2}+y_{d}^{2}\right)-\left(x_{0}^{2}+\right.\right.
$$

$\left.\mathrm{y}_{0}^{2}\right)$ ], which reduces the error [9]. After achieving the desired $r_{d}$ through the change in angle $C$ to angle $D$, angle $A$ is changed to $A^{\prime}$ to rotate the robot arm to reach the desired position. The pictorial representation is given in Figure 2.

Here, the rules are arranged as follows:

- For the position of Fig 2(a):

If(robot arm length needs to be changed by<fuzzy set $1>$, and current joint angle is <fuzzy set $2>$ ), then (change second link angle $\mathrm{C}$ by $\mathrm{E}$ )

- For the position of Fig 2(b):

If(change in angle $\mathrm{C}$ is $\mathrm{E}$, and desired angular change of robot arm length $T^{\prime}-T$ is <fuzzy set $3>$ ), then (change angle $A$ to $A^{\prime}$ ) where $<$ fuzzy set $i>(i=1,2$, ...) is of the form "positive big", "small" etc.

The developed fuzzy rule sets reside within the fuzzy controller, which outputs an incremental joint command to the individual joints of the robot based on the configuration and the deviations of the actual end point to the desired end point. The actual Cartesian end point is determined by applying the forward kinematic equations on joint angles $[10,11]$. The same procedure can be extended to 3 or higher DOF manipulators.

\section{Simulation}

The simulation of the proposed algorithm of the above algorithm, was done on a Mach operating system running NExT machine. The trajectory of a robot tracking a user specified straight line and partial configurations are shown in Figs. 3 and 4. The configurations of the robot are all in reasonable good positions, in the sense that those positions keep all joints away from their singular points. It also shows that the robot has passed one of its singular points, which usually causes an overflow in the conventional mathematical algorithm. The error between the actual and the desired trajectory are between specified limits. A computer simulation program is included in the Appendix.

Results of this simulation were graphed, and the performance for the position of the $x$ and $y$ co-ordinates and the error of the arm with respect to time were plotted (Figures $3 \& 4$ ). From Figure 3 one can see that the arm was successful in tracking the desired trajectory. Figure 4 shows that the error progressively decreases to zero in the least possible time.

\section{Conclusions}

A non algorithmic, model free approach has been developed that relies on a fuzzy rule base to evaluate the required axis motion for the robot. This scheme does not require solution to the inverse kinematic equation to arrive at the joint set points. The fuzzy rule base provides fast execution speed because the fuzzy rules 
perform simple integer additions and multiplications to evaluate the required axis motion. It can be shown that only a maximum of 15 rules are required to evaluate individual joint axis motion and that a linear relationship exists between the number of rules and the degree of freedom of the robot. The fuzzy logic controller approach is found to be $33 \%$ faster than traditional controller methods that require solution to the inverse kinematic equation. However, the fuzzy rule approach cannot achieve the tracking accuracies of the PID controller, since a single fuzzy rule describes a patch in the state space rather than an exact single point.

\section{References}

1. Zadeh, L. A. (1965). Fuzzy Sets. Information and control, 8:338-353.

2. T. Terano, K. Asai, M. Sugeno, "Fuzzy systems theory and its applications", Academic Press, Inc., San Diego, California, 1992.

3. J. J. Craig, "Introduction to Robotics, Mechanics and Control", Addison - Wesley Publishing Company, 1986, pp. 242-247.

4. H. Asada, J.J.E. Slotine, "Robot Analysis and Control", J. Wiley, New York, 1986.

5. L. A. Zadeh, "Outline of a New approach to the Analysis of Complex Systems and decision processes", IEEE Transaction Systems, Man and Cybernetics, Vol SMC-3, pp. 28-44, 1973.

6. A. Nedungadi, "A Fuzzy Robot Controller Hardware Implementation", IEEE International Conference on Fuzzy Systems, 1992.

7. "Applying Fuzzy Logic to Motion Control", Technology Today, Southwest Research Institute (SwRI), San Antonio, Texas, Sept. 1991.

8. N. J. Mandic, E. M. Scharf, and E. H. Mamdani, "Practical application of a heuristic fuzzy rule-based controller to the dynamic control of a robot arm", Proceedings of IEEE Part D, pp. 190-203, July 1985.

9. R. N. Lea, J. Hoblit and Y. Jani, "Fuzzy Logic Based Robotic Arm Control", Proceedings of the IEEE International Conference on Fuzzy Systems,San Francisco, California, March 28-April 1, 1993.

10. L. Sultan and T. Janabi, "The Cognitive bases for the design of a new class of fuzzy logic controllers", Proceedings of NAFIPS-92 International Conference, 1992.

11. L. Sultan and T, Janabi, "Intelligent Fuzzy Controller Development System For Handling Complex Control Problems", Canadian Conference on Electrical and Computer Engineering, 1992.

12. T. Yamkawa, "High speed fuzzy controller hardware system", Second Fuzzy Symposium, Japan, pp. 122-130, 1988.
13. G. V. S. Raju, J. Zhou, "Fuzzy Rule base approach for Robot Motion Control", IEEE International Conference on Fuzzy Systems, 1992.

\section{Anpendix}

$1^{*} \quad$ C program to compute the trajectory of the 2 DOF manipulator when the arm is constrained to move in a st. line of the form $y=-X+4 . * /$

\#define $\mathrm{m}-1 \quad f^{*}$ define the slope of the st. line $* /$ \#define y_intercept 4

\#define A $2 \%$ define a random $x$ value */

\#define B $2 \quad{ }^{*}$ define a y value for the first link */

\#define $\mathrm{C} 4 \quad l^{*}$ define the initial arm position */

\#include <stdio.h>

\#include <string.h>

double $x_{-}$final, $y_{-}$final;

double $x \_A, y \_B, x \_C[500], y \_D[500]$;

double dist 1 , dist2,armlenght, $\mathrm{D}$,arm 1_len;

FILE *fp;

main 0

1 double time[500];

double arm2_len, angle_2;

int $\mathrm{i}=0, \mathrm{j}$;

double error [500];

$\mathrm{D}=\left(\mathrm{m}^{*} \mathrm{C}\right)+\mathrm{y} \_$intercept;

fp = fopen("datafile","w");

$\operatorname{dist} 1=\operatorname{sqr} t(\operatorname{pow}(\mathrm{A}, 2)+\operatorname{pow}(\mathrm{B}, 2))$;

$\operatorname{dist} 2=\operatorname{sqrt}(\operatorname{pow}((A-C), 2)+\operatorname{pow}((B-D), 2))$;

armlenght $=\operatorname{sqrt}(\operatorname{pow}(\mathrm{C}, 2)+\operatorname{pow}(\mathrm{D}, 2))$;

puts("give the co-ordinates of final arm

position"):

scanf("\%d \%d", \&x_final, \&y_final);

arml_len = armlenght;

$x_{-} C[\bar{i}]=C ;$ time $[0]=0 ; y_{-} D[i]=D$;

error $[i]=0$;

do

( $\quad x_{-} C[i+1]=x_{-} C[i]+\left(C / a b s\left(x_{-}\right.\right.$final -

C));

y_intercept;

$$
y \_D[i+1]=m * x \_C[i+1]+
$$

pow $\left.\left(y_{-} D, 2\right)\right)$;

$$
\text { time }[\mathrm{i}+1]=\text { time }[\mathrm{i}]+0.1 \text {; }
$$

$$
++\mathrm{i} \text {; }
$$

\} while $\left(\left(\right.\right.$ error $[i-1]=a b s\left(x_{-} C[i-1]-x_{-}\right.$final $\left.)\right)<$ $0.01 \& \&$ abs $\left(y_{-} D[i-1]-y_{-}\right.$final $\left.)<0.01\right)$; for $(j=0 ; j<=i ;++j)$

fprintf(fp, "\%d ", time[j]); fprintf(fp, " \%d", error[j]); fprintf(fp, "\%d", $\left.x_{-} C[j]\right)$; fprintf(fp, " \%dn", y_D[j]); 


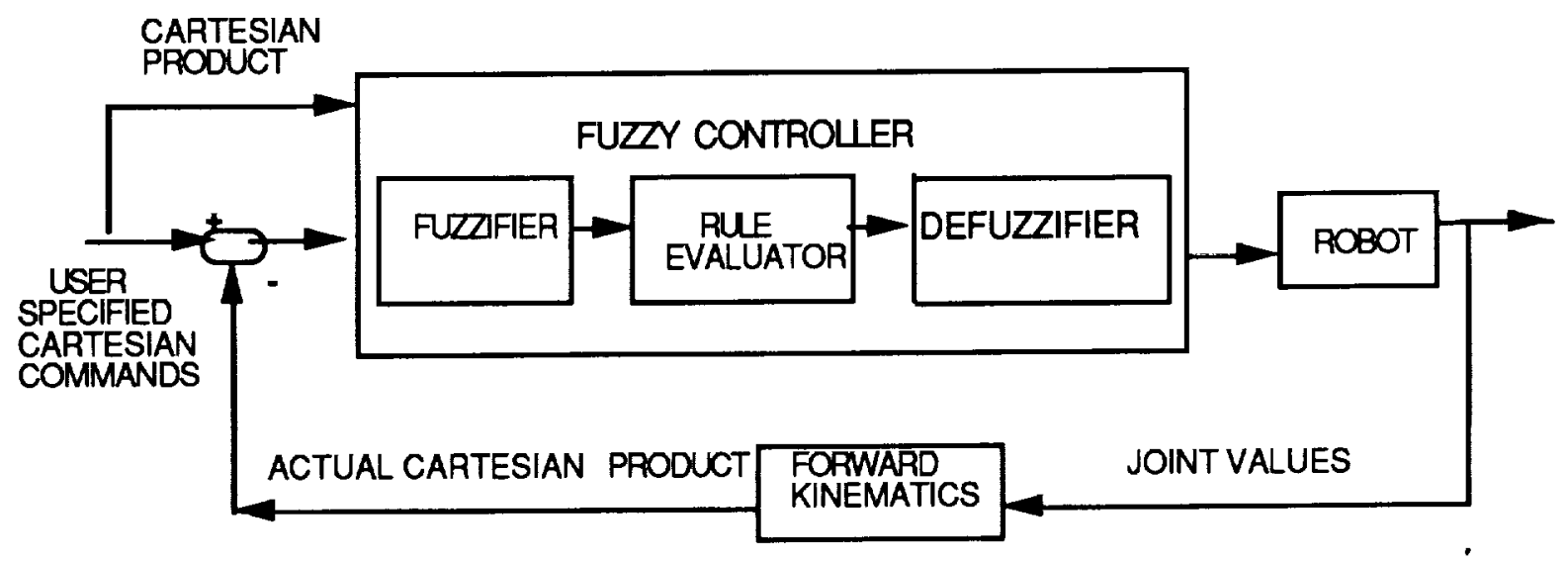

Figure 1. Block Diagram of Proposed Fuzzy Logic Controller

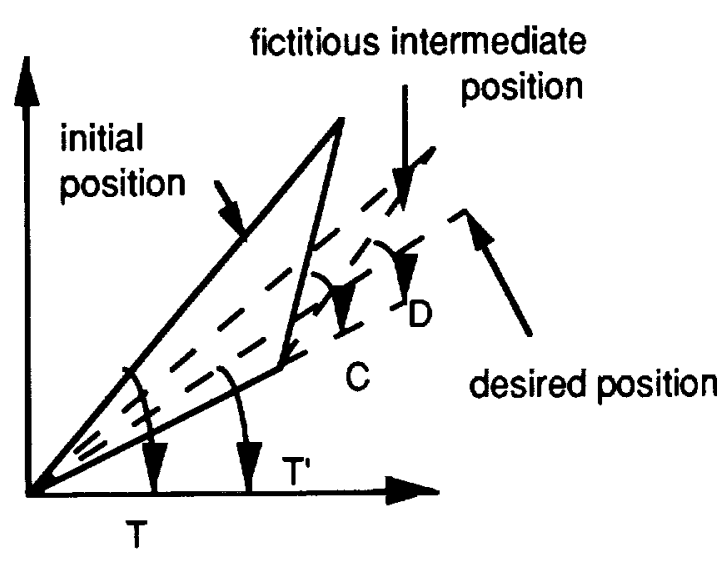

(a)

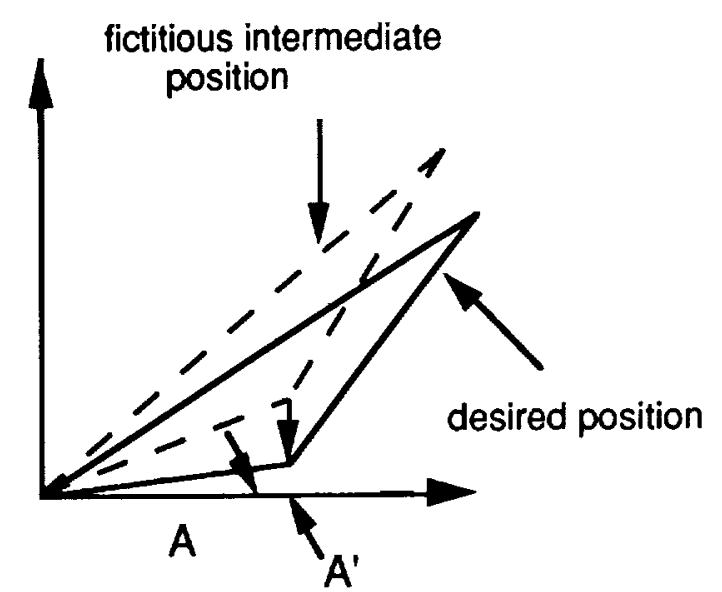

(b)

Figure 2. Stretching and Rotating the Robot Arm to Obtain Desired Position 
Fig 3: Graph of the $x$ Vs y co-ordinates of the manipulator, as it moves along the preset trajectory path, $y=-x+4$ (a straight line).
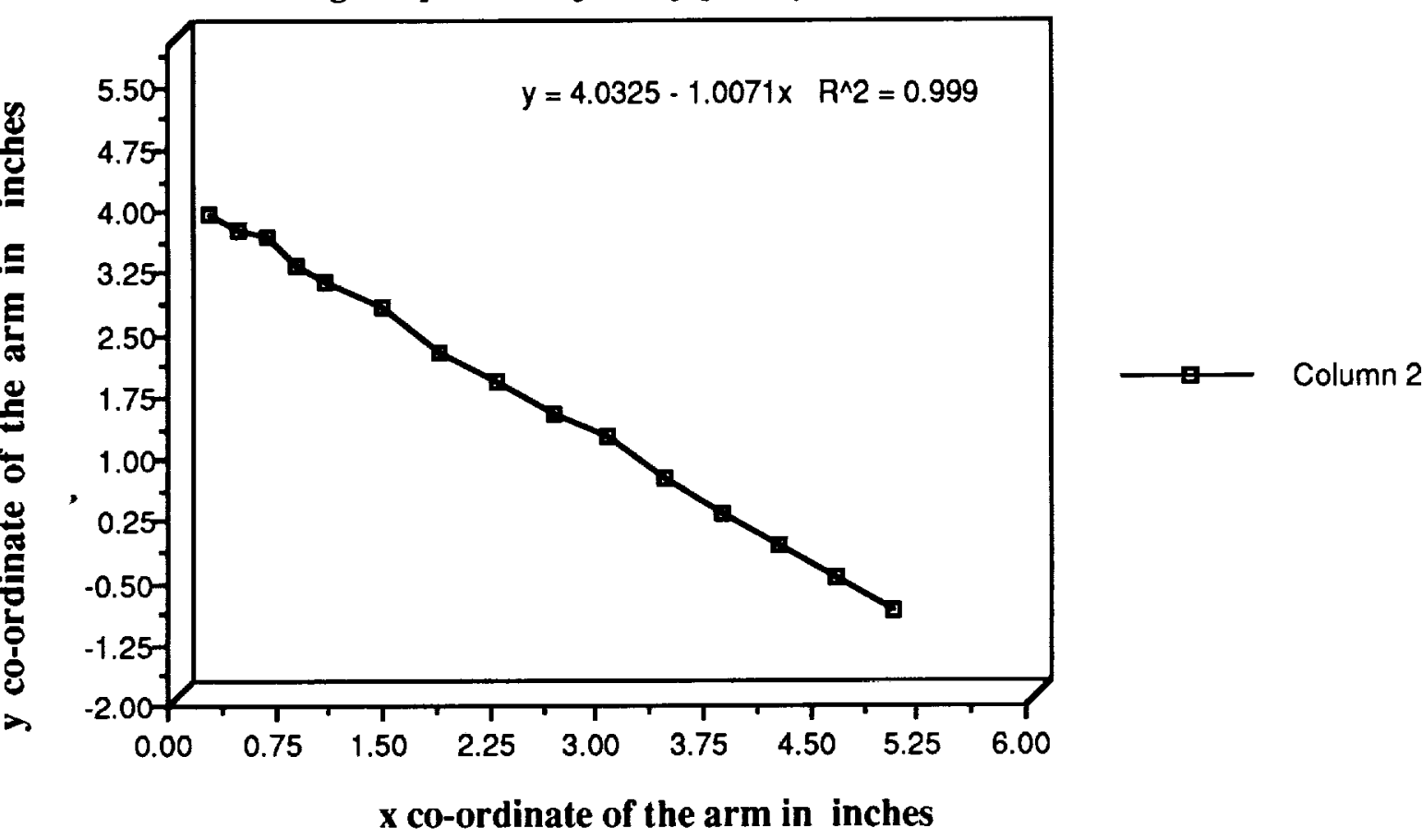

Fig 4: Graph of the error in the position of the manipulator at various time intervals in inches.

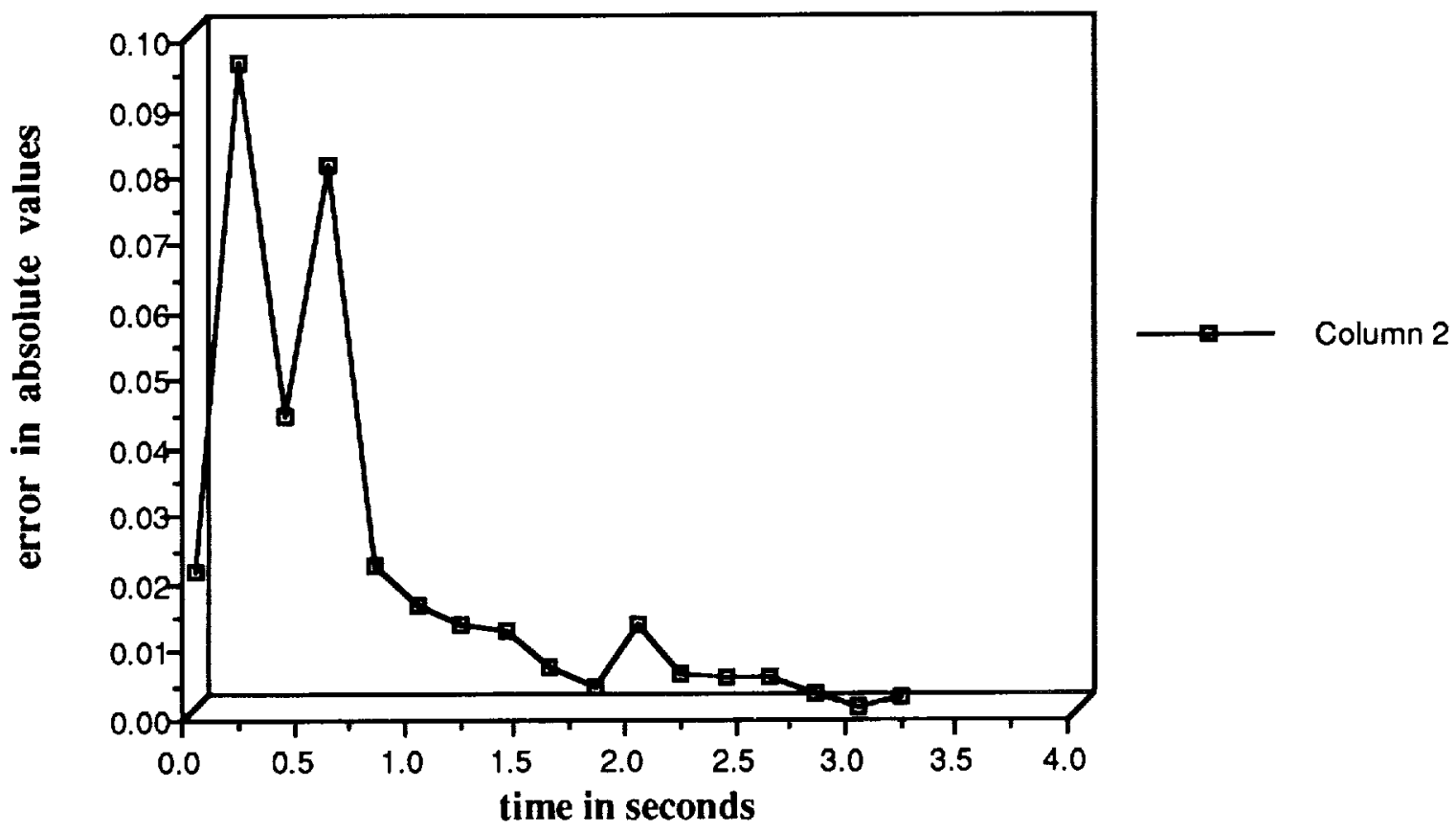

